\title{
The Early Development of Racial Bias in Infants and Childhood
}

\author{
Shuxian Meng \\ Syracuse University, Syracuse NY, United State
}

\begin{abstract}
Psychologists are interested in how humans process faces of their own-race and Otherraces, and there are plenty of previous research on this topic. This paper will summarize previous paper about Other-race Effect (ORE), and how do ORE develop racial bias in children. Researchers used Implicit Association Test (IAT) to assess implicit racial bias and found that implicit racial bias are different in different cultures and counties.
\end{abstract}

Keywords: Racial Bias; Other-race Effect; Implicit Association Test.

\section{Introduction}

Faces are important social markers in human life. How we process faces of own-race and Otherrace is a topic of intense interest for psychologists. The question has received extensive empirical investigation since the 1950s. The question that how children recognize and categorize faces of different races, and how such process might lead to later development of racial bias is discussed in recent literature.

\section{Racial Bias in Early Childhood}

\subsection{Other-race Effect}

It is now well established that individuals process faces from different races differently. One of the well-established phenomena is the Other-race Effect (Meissner\& Brigham, 2001). ORE refers to the phenomenon that human beings are easier to recognize their own races' faces than Other-race faces.

ORE develops early in human development. For example, researchers showed 3-, 6-, and 9-monthold Caucasian infants' pictures of African, Asian, Middle Eastern, and Caucasian faces, and recorded infants' attention to those faces (Kelly et al., 2007). It turned out that 6-month-old Caucasian infants can distinguish their own-race faces and Chinese faces, while 9-month-old Caucasian infants can only distinguish Caucasian faces from other races' faces (Kelly et al., 2007). The same phenomenon has been identified in other cultures, such as German. In Suhrke (2014), the researchers asked German children recognize faces of White women and African women, and the results suggest that the children can recognize their own race faces better (Suhrke et al., 2014).

\subsection{The Minimal Group Paradigm}

Recent evidence suggests that infants' facial recognition could possibly lead to children's racial bias. People tend to prefer others in their own groups, and this phenomenon can be observed at a really young age (Dunham, Baron \& Carey, 2011). Dunham, Baron, and Carey (2011) used different colors of coins to separate 33 children of 4 to 6-year-olds into groups, and let the kids rate the photos in different colors. This easy grouping method was enough to make them give higher rates to the photos that match their group colors (Dunham et al., 2011). This suggests that when people are sorted into a group, they show their preference to people of their own groups, and this is called the minimal group paradigm. In Raabe and Beelmann (2011)'s meta-analysis, they compared bias of kids from different age groups and found out that the peak of racial bias is when kids get to 5-7 years old. Afterwards, their racial bias decrease with increased age, from 8 to 10 years old. Children's prejudice change as they get older, and then remain stable throughout their puberty (Raabe\& Beelmann, 2011). 


\subsection{Implicit Association Test}

Past research on the development of racial bias has exclusively focused on explicit racial bias, a form of bias that is consciously aware of and is often measured via self-report. Another common type of racial bias that exists in children is implicit racial bias. Implicit racial bias refers to stereotypes or prejudices that are implicit, less conscious, and often unaware of (Greeenwald et al., 1995). One of the commonly used method to assess implicit racial bias is the Implicit Association Test, or IAT (Greenwald et al., 1998). IAT measures how closely two concepts are connected together in human minds. For example, in a standard IAT, the experimenter asks participants to press one key when they see white faces and good words or the other key for black faces and bad words. While participants respond accordingly, they response time and accuracy are recorded by the computer. After this combination block, the participants then are asked to respond in a reversed instruction: they are asked to press one key for black faces and good words or the other key for white faces and bad words. The logic of that IAT is that if participants respond slower and make more mistakes when they are asked to connect white and good, black and bad, it means they have implicit pro-white attitude, and vice versa (Greenwald, McGhee \& Schwartz, 1998).

Over the past 20 or so years, researchers have been adapting the IAT to assess children's implicit bias. For example, Baron and Banaji (2006) developed a child-friendly version IAT (Ch-IAT) to measure children's implicit racial bias. In the Ch-IAT, they added several child-friendly features, such as replace adult faces with children faces, use recoded voice instead of printed words, and used large Jellybean buttons instead of keys. Using this method, researchers have found that White children in the United State showed implicit pro-white biases (Baron \& Banaji, 2006; see similar results in White children in Canada, Steele et al., 2018). Specifically, the researchers found that White children of 6- and 10-years old reacted more rapidly when they saw White pictures associated with good words than when African American faces associated with good words (Baron \& Banaji, 2006). These evidences from Western literature suggest that implicit racial bias developed at least in childhood.

\subsection{Differences Across Countries}

Additionally, recent evidence further demonstrated implicit racial bias among Chinese children in China. Qian et al. (2016) further modified a preschooler-friendly version of IAT, which they referred to as the Implicit Racial Bias Test. They used smile and frown faces instead of two keys in traditional IAT tests. Using this method, Qian et al. (2016) measured implicit racial biases among 3 to 5 years old children in China (Qian et al., 2016). Among 139 Chinese kids that participated in their studies, they had negative implicit attitudes toward both Black and White and favor their own race (Qian et al., 2016). Even though the Cameroonian participants' implicit attitudes changed when they grow up to adulthood, their IAT results were similar with Chinese in their early age (Qian et al., 2016).

\section{Summary}

The researches above show that children can recognize their own-race faces better than Other-race races, and that they tend to prefer others in their own groups from a young age. The early emergence of perceptual bias in face processing may lead to later-on development of implicit racial bias in childhood.

\section{References}

[1] Baron, A. S., \& Banaji, M. R. (2006). The Development of Implicit Attitudes. Evidence of Race Evaluations from Ages 6 and 10 and Adulthood. Psychological Science, 17(1), 53-58. https:// Doi. org/ 10.1111/j.1467-9280.2005. 01664. X.

[2] Dunham, Y., Baron, A. S., \& Carey, S. (2011). Consequences of "Minimal" Group Affiliations in Children. Child Development, 82(3), 793-811. https://doi.org/10.1111/j.1467-8624.2011.01577.x. 
[3] Greenwald, A. G., \& Banaji, M. R. (1995). Implicit social cognition: Attitudes, self-esteem, and stereotypes. Psychological Review, 102(1), 4-27. https://doi.org/10.1037/0033-295x.102.1.4.

[4] Greenwald, A. G., McGhee, D. E., \& Schwartz, J. L. (1998). Measuring individual differences in implicit cognition: The implicit association test. Journal of Personality and Social Psychology, 74(6), 1464-1480. https: // doi.org/10.1037/0022-3514.74.6.1464.

[5] Kelly, D. J., Quinn, P. C., Slater, A. M., Lee, K., Ge, L., \& Pascalis, O. (2007). The Other-race Effect Develops During Infancy. Psychological Science, 18(12), 1084-1089. https://doi.org/10.1111/j.14679280.2007.02029.x.

[6] Meissner, C. A., \& Brigham, J. C. (2001). Thirty years of investigating the own-race bias in memory for faces: A meta-analytic review. Psychology, Public Policy, and Law, 7(1), 3-35. https:// doi.org/ 10. 1037/ 1076-8971.7.1.3.

[7] Qian, M. K., Heyman, G. D., Quinn, P. C., Messi, F. A., Fu, G., \& Lee, K. (2015). Implicit Racial Biases in Preschool Children and Adults from Asia and Africa. Child Development, 87(1), 285-296. https:// Doi. org/10.1111/cdev.12442.

[8] Raabe, T., \& Beelmann, A. (2011). Development of Ethnic, Racial, and National Prejudice in Childhood and Adolescence: A Multinational Meta-Analysis of Age Differences. Child Development, 82(6), 17151737. https://doi.org/10.1111/j.1467-8624.2011.01668.x.

[9] Steele, J. R., George, M., Williams, A., \& Tay, E. (2018). A cross-cultural investigation of children's implicit attitudes toward White and Black racial outgroups. Developmental Science, 21(6). https:// doi.org $/ 10.1111 /$ desc. 12673 .

[10] Suhrke, J., Freitag, C., Lamm, B., Teiser, J., Fassbender, I., Poloczek, S., Teubert, M., Vöhringer, I. A., Keller, H., Knopf, M., Lohaus, A., \& Schwarzer, G. (2014). The Other-race effect in 3-year-old German and Cameroonian children. Frontiers in Psychology, 5, 198. https://doi.org/10.3389/fpsyg.2014.00198. 